\title{
Molecular Mechanisms for the Optical Activities of Polyisocyanates Induced by Intramolecular Chiral Perturbations
}

\author{
Hong Gu, Takahiro Sato, Akio Teramoto, Lionel Varichon, and Mark M. Green* \\ Department of Macromolecular Science, Osaka University, Toyonaka, Osaka 560, Japan \\ * Department of Chemistry and Herman F. Mark Polymer Research Institute, Polytechnic University, \\ Six Metrotech Center, Brooklyn, N.Y. 11201, U.S.A.
}

(Received August 5, 1996)

\begin{abstract}
Molecular mechanisms were proposed to account for extraordinary optical activities of polyisocyanates containing chiral moieties (chiral monomer or initiator fragment) reported by Green et al. and by Okamoto et al., where the polyisocyanate molecule was modeled by a helical chain consisting of an alternating sequence of right-handed and left-handed helices with helix reversals in between. Theoretical predictions based on this model were consistent with remarkable dependence of optical rotation on monomer composition for random copolyisocyanates of chiral and achiral monomers and enantiomorphous copolymers and chain length dependence of optical rotation for achiral polyisocyanates with a chiral initiator fragment on the chain end. The helix reversal was shown to be the key factor for all the cases and more frequent for aromatic sidechain polymer poly $(m$-methyl phenyl isocyanate) than for aliphatic side chain polymers such as poly(butyl and hexyl isocyanate)s.

KEY WORDS Chiral / Optical Activity / Polyisocyanate / Helical Conformation / Helix Reversal / Statistical Mechanics / Copolymer /
\end{abstract}

Amide bonds of partial double-bond nature in a polyisocyanate chain tend to be coplanar, but they are distorted in a helical conformation due to steric interactions between the main chain and side chains. ${ }^{1-10}$ For this reason the global conformation of the polymer chain is well modeled by wormlike chains. ${ }^{7-9,11-18}$ However the sense of the helix is not determined for an achiral polyisocyanate because both the right-handed and lefthanded helices are symmetric. This symmetry is broken by a subtle chiral perturbation within a given molecule and this molecule becomes optical active. ${ }^{19-25}$ Indeed Green et al. ${ }^{20}$ showed that incorporation of a very small amount of chiral monomers into an achiral polyisocyanate in a random fashion gives rise to remarkable optical activity, which increases disproportionately with the mole fraction of chiral monomer. A similar disproportionate change in optical activity has been found in copolymers of enantiomorphous isocyanates $(R-S$ copolymers). ${ }^{25}$

On the other hand Okamoto and collaborators ${ }^{26,27}$ introduced a chiral perturbation into polyisocyanate chains in a different way; they synthesized polyisocyanates with chiral initiators, which were attached to a chain end, and showed that those polyisocyanates exhibited optical activity changing remarkably with length and temperature. The present study was motivated by these findings and tries to explore the molecular mechanisms behind the optical activities in these particular polymers. Green et al. ${ }^{20}$ called chiral entities sergeants and achiral entities soldiers. Thus we here deal with sergeants-soldiers polymers. Theoretically these polyisocyanates are regarded as typical linear cooperative systems just as chiral polyisocyanates and $\alpha$-helical polypeptides. Therefore we analyze their conformational properties including optical activity by the well-established statistical mechanical procedures based on the matrix method. ${ }^{28-30}$ A preliminary account was reported recently. ${ }^{31}$ Quite recently, Selinger and Selinger ${ }^{32}$ presented a theory of chiral order in random magnetic systems aiming at analyzing Green et al.'s data for enantiomorphous copolymers. They refer to the standard theory of random magnetic systems, which are special cases of linear Ising systems including those used for polypeptides $^{28-30}$ and polyisocyanates. ${ }^{21,33,34}$ As will be seen below, we will cover a wide variety of copolymers where the enantiomorphous copolymers are also included.

\section{THEORY}

Lifson et al. ${ }^{21}$ presented a statistical mechanical theory of the helical conformation of a chiral polyisocyanate, where the symmetry in helix sense is broken by the preference of a particular sense over the other due to the chiral perturbation from the chiral monomeric units, although the chiral effect is only minute on the monomer level but piled up greatly on a long chain. Their model consists of a polymer chain, where sequences of opposite helix senses appear alternatively with helix breaking points in between. There is a slight imbalance $\Delta G_{\mathrm{h}}$ in free energy between the units in the two helical conformations on the monomer level but the helix break costs a much larger energy $\Delta G_{\mathrm{r}}$. This is a typical linear Ising model problem and its theoretical basis has been established long since. ${ }^{28-30}$ Here we attempt to apply this theoretical treatment to our problem based on a model essentially the same as that of Lifson et al. Our polymer consists of $N$ monomeric units, each of which is either in the left-handed or right-handed helical conformation. Some of the units prefers a particular helix sense, and others do not; the latter may be achiral monomer units. According to the standard procedure in the linear Ising model calculation, ${ }^{28-30}$ the partition function $Z_{N}$ of the polymer consisting of $N$ monomeric units of type $i(k)$ may be expressed as 


$$
Z_{N}=\mathbf{A} \prod_{k=2}^{N} \mathbf{M}_{i(k)} \mathbf{B}
$$

where $\mathbf{M}_{i(k)}$ is the statistical weight matrix defined by

$$
\mathbf{M}_{i(k)}=\left[\begin{array}{cc}
u_{\mathrm{M}, i(k)} & u_{\mathrm{P}, i(k)} v_{i(k)} \\
u_{\mathrm{M}, i(k)} v_{i(k)} & u_{\mathrm{P}, i(k)}
\end{array}\right]
$$

with $u_{\mathrm{M}, i(k)}$ and $u_{\mathrm{P}, i(k)}$ being the statistical weights of the $k$-th monomer unit of type $i(k)$ in the left-handed and right-handed helices, respectively, and $v_{i(k)}$ the transition probability from the left-to-right or right-to-left helices. For the sake of simplicity, hereafter the notation $i(k)$ is abbreviated as $i$. These statistical weights are related to the free energies defined above by

$$
\begin{aligned}
u_{\mathrm{M}, i} & =\exp \left[+\Delta G_{\mathrm{h}, i}(T) / R T\right] \quad u_{\mathrm{P}, i}=1 / u_{\mathrm{M}, i} \\
v_{i} & =\exp \left[-\Delta G_{\mathrm{r}, i}(T) / R T\right]
\end{aligned}
$$

Here $\mathbf{A}$ and $\mathbf{B}$ are the terminal vectors specifying the states of the terminal units defined by

$$
\mathbf{A}=\left(a u_{\mathrm{M}, i} u_{\mathrm{P}, i}\right) \quad \mathbf{B}=\left(\begin{array}{l}
b \\
1
\end{array}\right)
$$

where $a$ and $b$ measure the relative preference of the right-handed helix in the first and final monomer units, respectively. The average number $n_{\mathrm{M}}$ of monomer units in the right-handed helical conformation is calculated from

$$
n_{\mathrm{M}}=\sum_{i} \frac{\partial\left(\ln Z_{N}\right)}{\partial\left(\ln u_{\mathrm{M}, i}\right)}
$$

In a similar way the average number of helix reversals in a chain $n_{\mathbf{R}}$ is calculated from

$$
n_{\mathrm{R}}=\sum_{i} \frac{\partial\left(\ln Z_{N}\right)}{\partial\left(\ln v_{i}\right)}
$$

On the assumption that only helical conformations contribute to optical activity, the specific rotation $[\alpha]$ is related to $n_{\mathrm{M}}$ or the fraction of units in the M-helix $f_{\mathrm{M}}=n_{\mathrm{M}} / N$ by

$$
[\alpha]=[\alpha]_{\mathrm{m}}\left(n_{\mathrm{M}} / N\right)-[\alpha]_{\mathrm{m}}\left(N-n_{\mathrm{M}}\right) / N=[\alpha]_{\mathrm{m}}\left(2 f_{\mathrm{M}}-1\right)
$$

where $[\alpha]_{\mathrm{m}}$ is the value of $[\alpha]$ for perfect M-helix.

Actually Lifson et al. ${ }^{21}$ treated one of the simplest cases where there is only one kind of monomeric unit but with non-zero $\Delta G_{\mathrm{h}}$. We are here concerned with two particular types of polymers, which correspond to the two types of chiral perturbation on the conformation of polyisocyanates experimentally studied: (1) random copolymers of two monomeric units, one of which is chiral and the other is not or of different chirality and (2) polymers undergoing chiral perturbation only from the chain end(s).

\section{Type 1 Polymers}

For this polymer we have one unit $(i=1)$ with $u_{\mathbf{M}, 1}$ $>u_{\mathrm{P}, 1}\left(=1 / u_{\mathrm{M}, 1}\right)$ and the other unit $(i=2)$ with $u_{\mathrm{M}, 2}$ $=u_{\mathrm{P}, 2}=1$ for sergeant-soldier copolymers and $u_{\mathrm{M}, 2}$ $=1 / u_{\mathrm{M}, 1}$ and $u_{\mathrm{P}, 2}=1 / u_{\mathrm{P}, 1}$ for enantiomorphous copolymers and for simplicity we assign the same small value $v(\ll 1)$ to $v$ of both units $\left(v_{1}=v_{2}=v\right) ; a=b=1$. Let us start with discussing a sample consisting of polymer species of a fixed chain length $N$ and a given monomer composition $x$ or number of chiral units $p$ but distributed randomly on each chain; $x=p / N$. Thus this sample is referred to as a uniform random copolymer with the specific rotation $[\alpha](N, p)$ of a function of $N$ and $p$. For this type of polymer, the average number of units in the right-handed helices $n_{\mathrm{M}}$ is given by

$$
n_{\mathrm{M}}=\left\langle\sum_{i=1}^{2} \frac{\partial\left(\ln Z_{N}\right)}{\partial\left(\ln u_{\mathrm{M}, i}\right)}\right\rangle
$$

and the average number of helix reversals $n_{\mathrm{R}}$ is given by

$$
n_{\mathrm{R}}=\left\langle\sum_{i=1}^{2} \frac{\partial\left(\ln Z_{N}\right)}{\partial\left(\ln v_{i}\right)}\right\rangle
$$

where $\langle>$ means the average of a given quantity over polymer species with random sequence in the given sample. It is easy to extend this treatment to polymers consisting of two or more types of chiral units.

Generally speaking, however, a given sample can be heterogeneous with respect to $N$ and $p$ or to both, which is referred to as statistical copolymer. Let $\omega(N, p)$ be the mole fraction of a polymer species with $N$ and $p$. Then the average optical rotation $[\bar{\alpha}]$ of this sample is expressed by

$$
[\bar{\alpha}]=\sum_{N} \sum_{p=0}^{N}[\alpha](N, p) M_{\mathrm{res}} N \omega(N, p) / \sum_{N} \sum_{p=0}^{N} M_{\mathrm{res}} N \omega(N, p)
$$

with

$$
\sum_{N} \sum_{p=0}^{N} \omega(N, p)=1
$$

where $[\alpha](N, p)$ is the specific rotation of a uniform random copolymer with $N$ and $p$ and $M_{\text {res }}=(1 / N)\left[M_{1} p+\right.$ $\left.(N-p) M_{2}\right]$, with $M_{1}$ and $M_{2}$ are the molar masses of chiral and achiral monomer units, respectively.

Actual calculations were performed by using eq 7 through eq 9 with appropriate values chosen for $u_{\mathbf{M}, 1}$ $\left(=u_{\mathrm{M}}\right), v$, and $[\alpha]_{\mathrm{m}}$ for appropriately generated random copolymers and taking the averages. The results were also used with eq 10 along with an appropriate $\omega(N, p)$ to calculate $[\bar{\alpha}]$ for statistical copolymers. It is important to note that in all these calculations, we assumed that $\mathbf{M}_{i}$ depends on the type of residue $i$ but not on its location on the chain. This assumption would be accurate when the sergeant residues are small in fraction and dispersed isolated among the soldier residues or in the opposite case. Selinger and Selinger ${ }^{32}$ have treated enantiomorphous copolymers and given similar arguments.

\section{Type 2 Polymers}

The $Z_{N}$ of a polymer with chiral moieties at the chain end(s) is expressed by an analytical expression:

$$
Z_{N}=\sum_{k= \pm} X_{k} \lambda_{k}^{(N-1)} Y_{k}
$$

where $\lambda_{k}(k= \pm)$ are the eigenvalues of the matrix $\mathbf{M}$ :

$$
\lambda_{ \pm}=\frac{1}{2}\left[u_{\mathrm{M}}+u_{\mathrm{P}} \pm \sqrt{\left(u_{\mathrm{M}}-u_{\mathrm{P}}\right)^{2}+4 u_{\mathrm{M}} u_{\mathrm{P}} v^{2}}\right]
$$


and $X_{k}$ and $Y_{k}$ are defined, respectively, by

$$
X_{k}=a u_{\mathrm{M}}+b U_{2}\left(\lambda_{k}\right) \quad Y_{k}=b V_{1}\left(\lambda_{k}\right)+V_{2}\left(\lambda_{k}\right)
$$

with $U_{j}$ and $V_{j}(j=1,2)$ are the components of the lefthand and right-hand eigenvectors $\mathbf{U}$ and $\mathbf{V}$, respectively, whose components are given by

$$
\begin{array}{ll}
U_{1}=1 & U_{2}=\left(\lambda-u_{\mathrm{M}}\right) /\left(u_{\mathrm{P}} v\right) \\
V_{1}=\left(\lambda-u_{\mathrm{P}}\right) /\left(2 \lambda-u_{\mathrm{M}}-u_{\mathrm{P}}\right) & V_{2}=u_{\mathrm{P}} v /\left(2 \lambda-u_{\mathrm{M}}-u_{\mathrm{P}}\right)
\end{array}
$$

For a polymer with a chiral initiator only at one end, $a>1$ and $b=1$, whereas both $a$ and $b$ differ from unity for a polymer with chiral moieties at both ends. The average number of units in the right-handed (or lefthanded) helices is calculated by eq 5 with eq 12 and its analytical expression reads

$$
\begin{aligned}
n_{\mathrm{M}}= & \frac{1}{Z_{N}} \sum_{k= \pm} u_{k}\left[X_{k,(\mathrm{M})} \lambda_{k}^{(N-1)} Y_{k}\right. \\
& \left.+(N-1) X_{k} \lambda_{k,(\mathrm{M})} \lambda_{k}^{(N-2)} Y_{k}+X_{k} \lambda_{k}^{(N-1)} Y_{k,(\mathrm{M})}\right]
\end{aligned}
$$

where the subscript (M) signifies differentiation with respect to $u_{\mathbf{M}}$. The number of helix reversals is derived in a similar way as

$$
\begin{aligned}
n_{\mathrm{R}}= & \frac{1}{Z_{N}} \sum_{k= \pm} v\left[X_{k,(\mathrm{R})} \lambda_{k}^{(N-1)} Y_{k}\right. \\
& \left.+(N-1) X_{k} \lambda_{k,(\mathrm{R})} \lambda_{k}^{(N-2)} Y_{k}+X_{k} \lambda_{k}^{(N-1)} Y_{k,(\mathrm{R})}\right]
\end{aligned}
$$

where the subscript $(\mathrm{R})$ refers to the differentiation with respect to $v$. For simplicity, we have assumed that the residue molecular weights are the same for the chiral moieties and the monomer unit.

\section{NUMERICAL RESULTS}

\section{Type 1 Polymers}

For type 1 polymers, we first consider the effect on optical rotation of such factors as the chain length $N$, the fraction of the sergeant monomers $x$, the strength of the sergeant monomer $u_{\mathrm{M}}$, and the helix reversal probability $v$, where $u_{\mathrm{M}}$ is related to the residue free energy difference in the right-handed and left-handed helical conformations $\Delta G_{\mathrm{h}}$ by $u_{\mathrm{M}}=u_{\mathrm{M}, 1}=\exp \left(-\Delta G_{\mathrm{h}} / 2 R T\right)$ and $v$ is related to the free energy of the reversal residue $\Delta G_{\mathrm{r}}$ by $v=\exp \left(-\Delta G_{\mathrm{r}} / R T\right)$. For the soldier monomers, $u_{\mathrm{M}, 2}=u_{\mathrm{P}, 2}=1$. Here $a=b=1$.

Figure 1 shows relations between $2 f_{\mathrm{M}}-1$ and $N$ for uniform random copolymers with a fixed composition $x=p / N$; note that $2 f_{\mathrm{M}}-1=[\alpha](N, p) /[\alpha]_{\mathrm{m}}$. The panel (a) gives $2 f_{\mathrm{M}}-1$ with $v=0.005$ and $x=0.1$ and different $u_{\mathrm{M}}$ values and panel (b) gives those with $u_{\mathrm{M}}=1.1$ and $x=0.04$ and different $v$ values; each $2 f_{M}-1$ value is the average over $10-20$ randomly generated chains; further increase in sampling number had no significant effect. In all cases the $N$ dependence of $2 f_{\mathrm{M}}-1$, when plotted double-logarithmically, is represented by a sigmoidal curve but the shape of the curve varies with different combinations of the parameters $u_{\mathrm{M}}$ and $v$. It can be seen in panel (a) that $2 f_{\mathrm{M}}-1$ is larger for larger $u_{\mathrm{M}}$, namely with larger chiral perturbation. On the other hand, the panel (b) shows that as $v$ is increased with a given value of $u_{\mathrm{M}}$ it becomes smaller and the curve becomes less

Polym. J., Vol. 29, No. 1, 1997

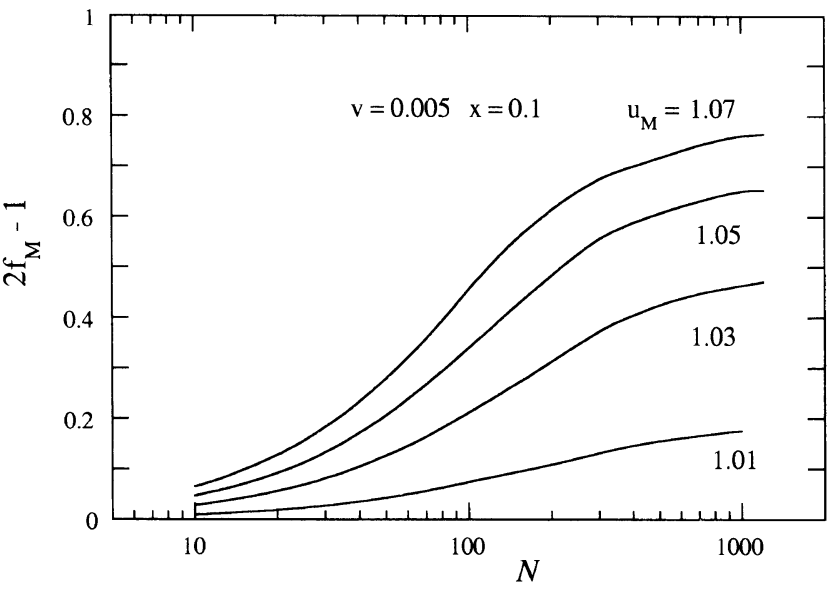

(a)

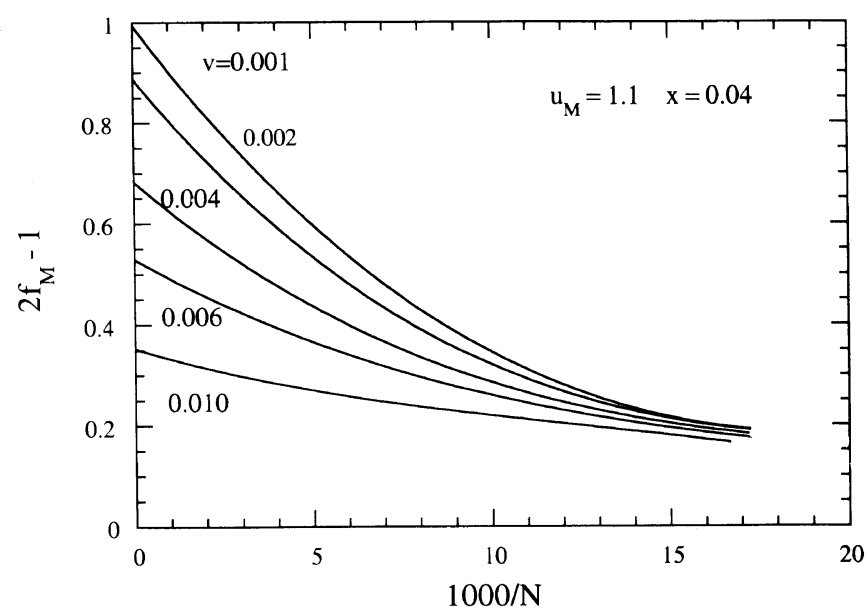

(b)

Figure 1. Plots of $2 f_{\mathrm{M}}-1$ against $N$ for uniform random copolymers of $x=0.1$. Panel (a), $v=0.005, x=0.1$ and various $u_{\mathrm{M}}$ values; panel (b), $u_{\mathrm{M}}=1.06, x=0.04$ and different $v$ values. $2 f_{\mathrm{M}}-1=[\alpha](N, x) /[\alpha]_{\mathrm{m}}$.

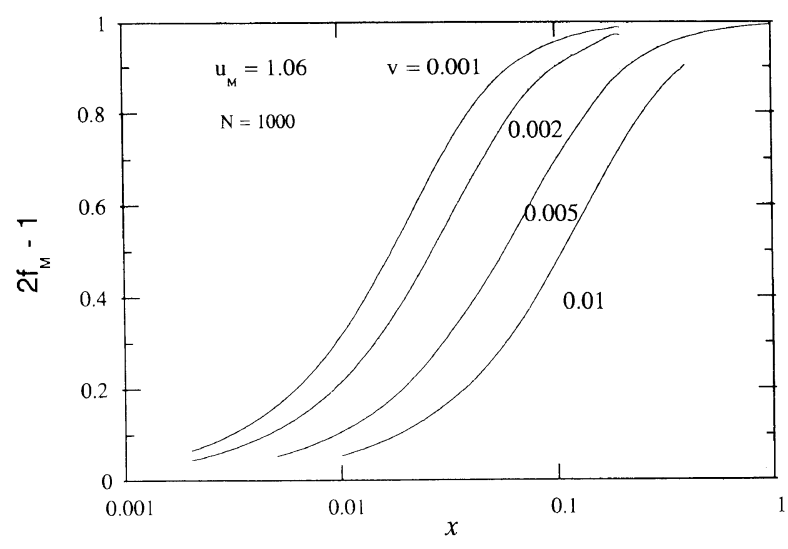

Figure 2. Dependence of $2 f_{\mathrm{M}}-1$ on $x$ for uniform random copolymers of $N=1000$ with $u_{\mathrm{M}}=1.06$ and different $v$ values.

steep and moves to larger $N$. Thus we see that the $N$ dependence of $2 f_{\mathrm{M}}-1$ is determined essentially by $v$, namely the helix reversal probability, whereas both $u_{\mathrm{M}}$ and $v$ contribute to its magnitude. This is understood from the finding that when plotted double-logarithmically, all the curves with the same $v$ value have substantially the same shapes.

Figure 2 illustrates how $2 f_{\mathrm{M}}-1$ of a polymer with $N=1000$ depends on $\log x$ for the values of $u_{\mathrm{M}}$ and $v$ 
indicated. It can be shown that $v$ and $u_{\mathrm{M}}$ play similar roles as in Figure 1. Indeed we see that the $x$ dependence of $2 f_{\mathrm{M}}-1$ is governed by $v$, whereas its magnitude depends on both $u_{\mathrm{M}}$ and $v$. Figure 1 combined with Figure 2 indicates that the $2 f_{\mathrm{M}}-1$ vs. $N$ curve is shifted to larger $N$ as $x$ is decreased. However it is noted that a very small chiral perturbation, the presence of the chiral monomer by $1 \%$, induces a significant optical rotation with appropriate parameter values, and this induction is more conspicuous with smaller $v$, namely when the helix reversal is more difficult to occur. Recently Selinger and Selinger ${ }^{32}$ studied conformations and of enantiomorpous copolymers and revealed interesting qualitative conformational features. Their results, when transcribed to chiral-achiral copolymers, are in accord with our above discussions.

Next we examine effects of composition heterogeneity on $[\alpha]$ for a polymer sample, which consists of chains of a uniform length $N$ but of different $p$, assuming a particular distribution function

$$
\omega(N, p)=x^{p}(1-x)^{N-p}\left(\frac{N !}{(N-p) ! p !}\right)
$$

where $p$ is the number of chiral units in a particular chain, with the average number of chiral units $\langle p\rangle$ given by

$$
\langle p\rangle=N x
$$

In other words, the average mole fraction of chiral unit is $x=\langle p\rangle / N$. The contribution of a polymer species with $N$ and $x$ to the total optical activity is given by $[\alpha](N, p) \omega(N, p) /[\alpha]_{\mathrm{m}}$ for $M_{1}=M_{2}=M_{0}$, where $M_{1}$ and $M_{2}$ are the molar masses of the chiral and achiral monomer units, respectively.

Figure 3 shows plots of $[\alpha](N, p) \omega(N, p) /[\alpha]_{\mathrm{m}}$ vs. $p$ for different $N$ with $x=0.1$. The curve for each $N$ has a sharp maximum located around $p=N x$. For a polymer monodisperse in $N$, the area under each curve is proportional to the average optical rotation of the polymer $[\bar{\alpha}](N, x)$ :

$$
[\bar{\alpha}](N, x)=\sum_{p=0}^{N}[\alpha](N, p) M_{\mathrm{res}} \omega(N, p) / \sum_{p=0}^{N} M_{\mathrm{res}} \omega(N, p)
$$

It was found that $[\bar{\alpha}](N, x)$ is close to $[\alpha](N, p)$ for

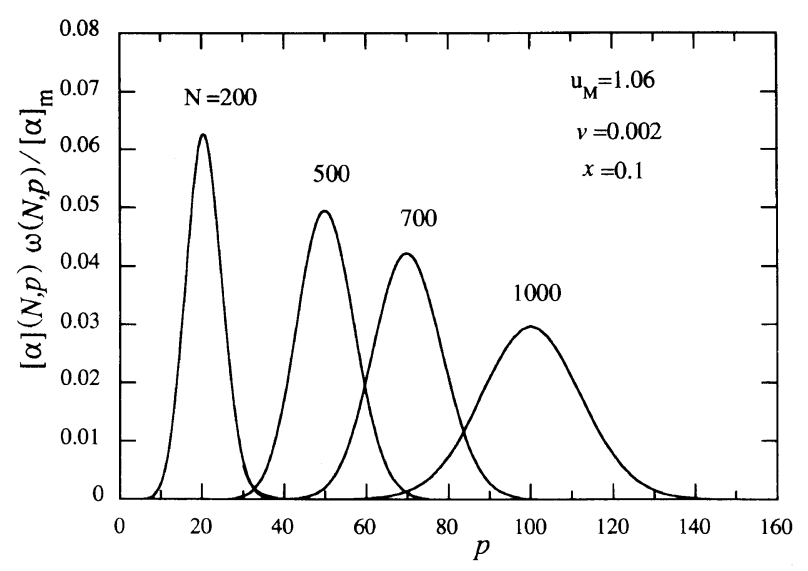

Figure 3. Plots of $[\alpha](N, x) \omega(N, p) /[\alpha]_{\mathrm{m}}$ against the number of chiral residues $p$ for the chains of different lengths $N$. Parameters: $u_{\mathrm{M}}=1.06$, $v=0.002, x=0.1$. various sets of the parameter values, $u_{\mathrm{M}}, v, x$, and $N$ as far as the distribution eq 18 is assumed. Therefore experimental $[\alpha]$ for narrow-distribution samples may be equated to $[\alpha](N, p)$ to a good approximation. On the other hand, the heterogeneity in $N$ has complicated effects on the average conformational properties of the sample and no explicit relation is derived unless the detailed knowledge of the heterogeneity is available. However in the limit of very large $N$ where $N v>3$, the following asymptotic relation is expected:

$$
[\bar{\alpha}] /[\alpha]_{\mathrm{m}}=K_{1}-K_{2} / N_{n}
$$

where $K_{1}$ and $K_{2}$ are numerical constants dependent on $u_{\mathrm{M}}, v$, and $x$, and $N_{n}$ is the number average chain length of the sample.

\section{Type 2 Polymers}

Next we consider type 2 polymers, which receive chiral perturbations from one chain end or both ends. The chiral moiety (sergeant) develops a particular helical sense, which propagates along the chain, giving rise to significant optical activity. However this helical conformation is reversed by helix reversal, reducing the optical activity. Indeed average conformational properties including optical activity are determined by the strength of the chiral perturbation of the chiral fragment(s) $a$ (and $b$ ) and the helix reversal probability $v$ along with $N$.

Figure 4 illustrates how $[\alpha]$ varies with $N$ for a polymer with a chiral fragment at one end with various values of $a$ and $v$ and $b=1$. As naturally expected, a stronger sergeant (a large $a$ ) forces the soldiers to obey his command more strictly, yielding larger $[\alpha]$, but his command diminishes rapidly with increasing number of soldiers. Indeed in each case $[\alpha] /[\alpha]_{\mathrm{m}}$ starts with $2 a /$ $(a+1)-1=(a-1) /(a+1)$ at $N=1$, decreases with increasing $N$ to vanish at infinite $N$. The circles on the $a=9$ curves represent the values with $a=2$ multiplied by $12 / 5$, which appear plotted on the curves with $a=2$ with the same $v$ values. This indicates that for $b=1$ the molecular weight dependence is almost unchanged with the value of $a$, although the magnitude of $[\alpha]$ changes largely. It can be shown that for $b=1$ the $[\alpha] /[\alpha]_{\mathrm{m}}$ divided by $(a-1) /(a+1)$ is a function only of $v$ and $N$. Panel (b) of Figure 4 shows the dependence of $n_{\mathrm{R}}$ on $N$ for different $v$ values. It can be seen in both panels that the role of $v$ in $[\alpha]$ is important at all $N$. For example, with $v=0.010$, helix reversal occurs on one chain among five chains with $N$ as small as 20 and $n_{\mathrm{R}}$ exceeds 1 above $N=103$.

The chiral perturbation from both ends changes the $N$ dependence significantly as shown in Figure 5, depending on whether the two commanders are allies or not. The allied commanders $(b=9)$ cooperate with each other to demand stricter control of the soldiers, while the control is weakened in the opposite case $(b=0.2)$. It is also seen that the cooperation is strengthened for smaller $v(0.001)$. No experimental data to test this prediction has as yet been reported.

\section{ANALYSIS OF EXPERIMENTAL DATA}

Green et al. ${ }^{20}$ reported data for $\mathrm{Na} \mathrm{D}$-line specific 


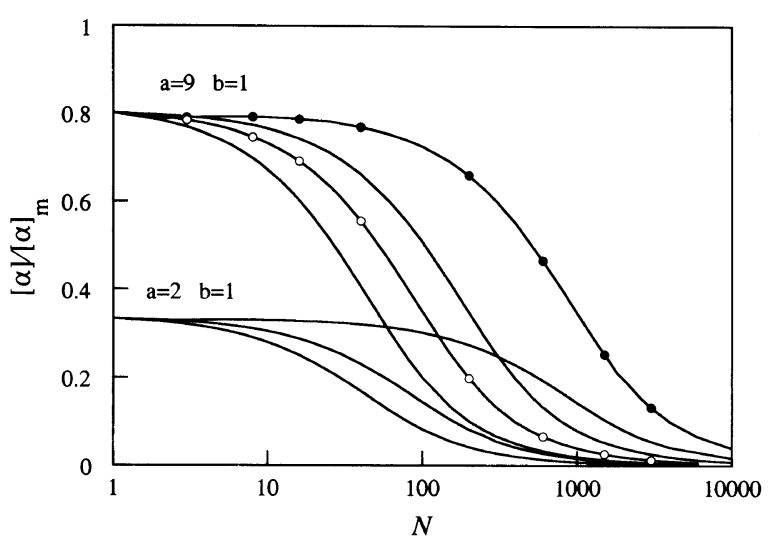

(a)

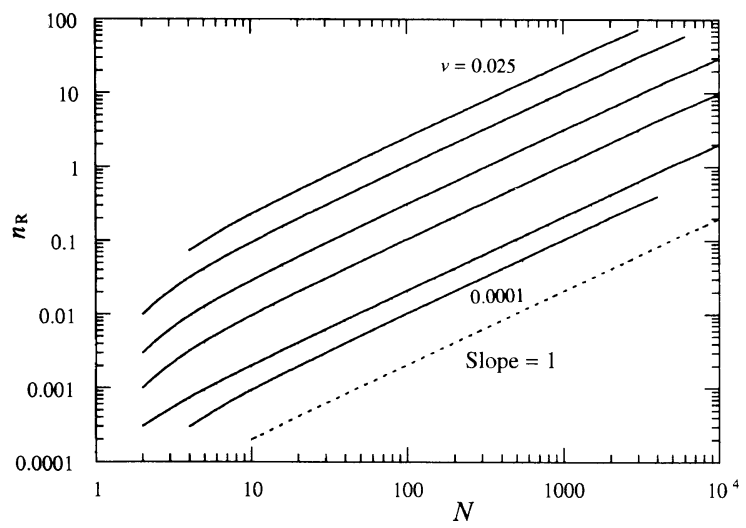

(b)

Figure 4. Conformation and optical activity of polymers with a chiral fragment at one chain end. Panel (a), plots of $[\alpha] /[\alpha]_{\mathrm{m}}$ against $N$ of polymers with a chiral fragment at one chain end with $\alpha=9$ and 2 and various values of $v$. For $a=9$ and $b=1, v=0.001,0.005,0.02$, and 0.01 from top to bottom, and for $a=2$ and $b=1, v=0.001,0.01$, and 0.02 from top to bottom; panel (b), number of helix reversals per chain for different $v$ of $0.025,0.01,0.003,0.001,0.0002$, and 0.0001 from top to bottom.

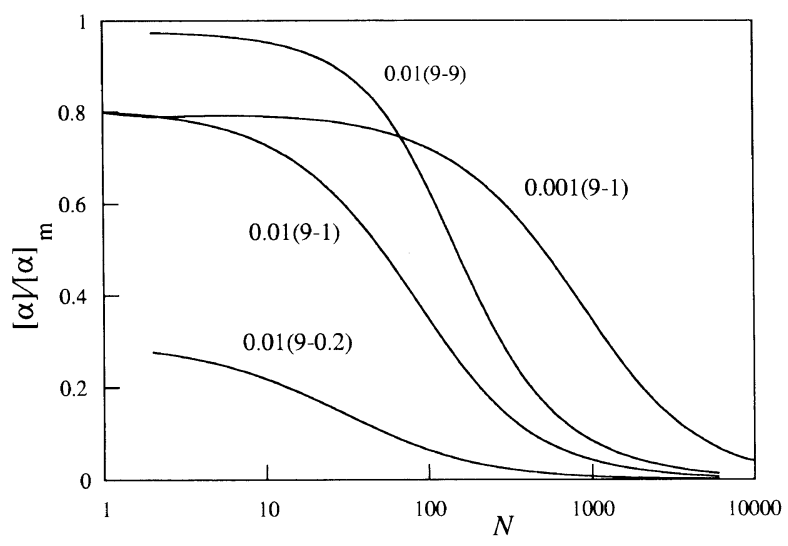

Figure 5. Plots of $[\alpha] /[\alpha]_{\mathrm{m}}$ against $N$ for polymers with chiral fragments at both chain ends for different combinations of $v(a-b)$.

rotation $[\alpha]_{\mathrm{D}}$ for copolymers of $(R)$-2,6-dimethylheptyl isocyanate (NIC) and hexyl isocyanate (HIC) of various monomer compositions $x$ in chloroform. It was shown that each polymer consisted of random arrangements of

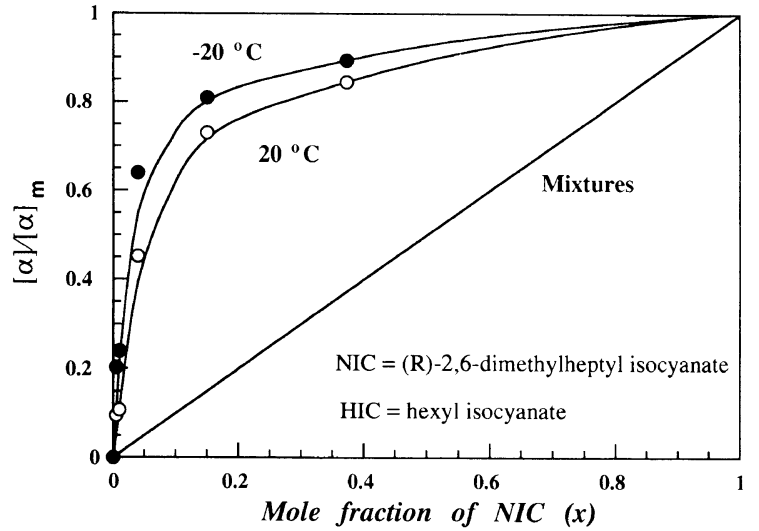

(a)

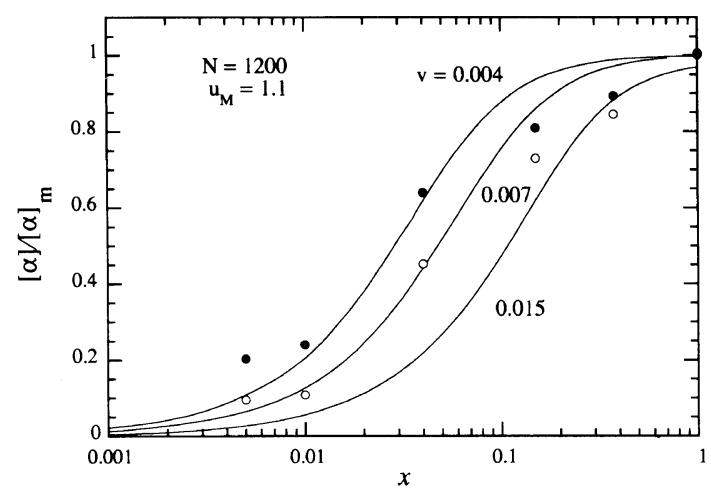

(b)

Figure 6. Dependence of $[\alpha] /[\alpha]_{\mathrm{m}}$ for random copolymers of chiral and achiral monomers on the fraction of chiral monomer $x$. Panel (a), experimental data of Green et al..$^{20}$ for copolymers of NIC and HIC: open circles, $20^{\circ} \mathrm{C}$ and filled circles, $-20^{\circ} \mathrm{C}$; curves; panel (b) theoretical values for $N=1200$ calculated for uniform random co polymers using the parameter values indicated.

the chiral and achiral monomer units forming very long chains and thus it can be regarded approximately as a uniform random copolymer defined in Theory section. Figure $6(\mathrm{a})$ shows their data, where $[\alpha]_{\mathrm{D}}$ increases sharply at small $x$, indicating that NIC is a commander exerting a moderate chiral perturbation. These data should be compared with theoretical $[\bar{\alpha}](N, x)$, which are replaced in panel (b) by $[\alpha](N, p)$ for the reason discussed in the previous section. Thus the curves in the figure represent theoretical values for uniform random copolymers calculated by the matrix method as explained above, $[\alpha](N, p)$. The data at $20^{\circ} \mathrm{C}$ (unfilled circles) are roughly fitted to the curve with $u_{\mathrm{M}}=1.1$ and $v=$ 0.007 , excepting that the two data points at higher $x$ deviate downward from the curve. ${ }^{* 1}$ Trial-and-error calculations revealed that $u_{\mathrm{M}}$ would be in the range $1.06-1.2$ and $v$ in the range $0.004-0.015$. The central value 0.007 of $v$ may be compared with 0.004 obtained previously for poly $((R)-i$-deuterio- $n$-hexyl isocyanate) $(i=1,2)$ in dichloromethane at $20^{\circ} \mathrm{C}$ (see Table I). ${ }^{33,34}$ Thus we take the results shown in Figure 6 to be consistent with the assumed molecular mechanism. This $v$ value implies that there exists one chain with a helix

\footnotetext{
*1 If it is assumed that optical rotation arises only from the helical conformation of the main chain, it is regitimate to use mean residue rotation ( $[\alpha]$ based on the mass concentration times $M_{\mathrm{res}}$ ) as a measure for $2 f_{\mathrm{M}}-1$. However it is not clear whether this assumption is strictly valid or the side chain has some effect on $[\alpha]$. In view of this situation, we here use $[\alpha]$ without being corrected for $M_{\text {res. }}$.
} 
Table I. Statistical thermodynamic parameters for chiral polyisocyanates

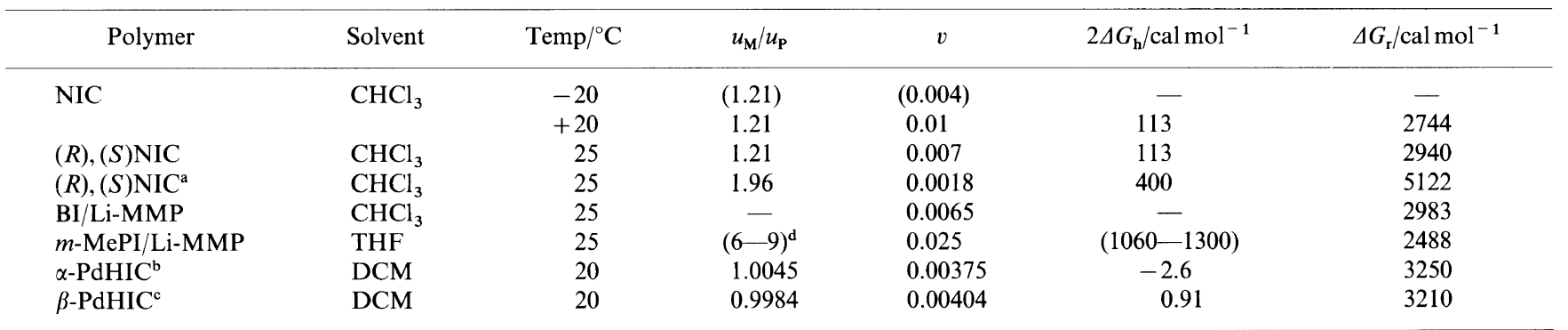

${ }^{a}$ Values calculated from Selinger and Selinger. ${ }^{32}{ }^{\mathrm{b}}$ Taken from ref $33 .{ }^{\mathrm{c}}$ Taken from ref $34 .{ }^{\mathrm{d}}$ Rough estimates of $a$. ( ) Values in the parentheses are not accurate.

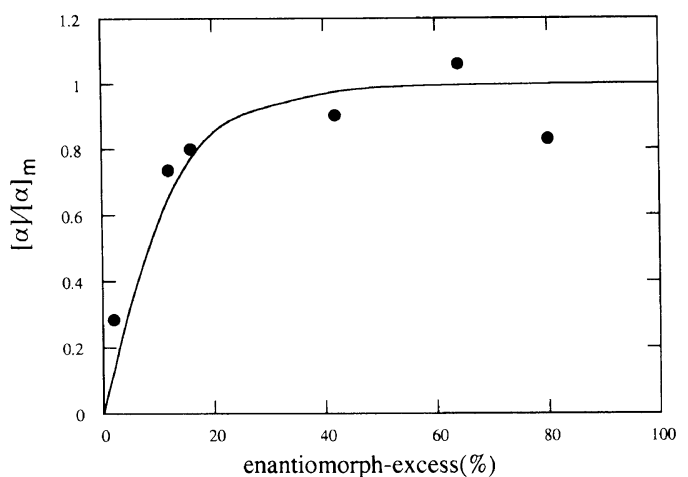

Figure 7. Dependence of $[\alpha] /[\alpha]_{\mathrm{m}}$ for random copolymers of $(R)$ and $(S)$-2,6-dimethylheptyl isocyanates on the enantiomorphous excess $(\%)$ of $(R)$-2,6-dimethylheptyl isocyanate. Circles, experimental data of Green et al. ${ }^{25}$; curves, solid curve, theoretical values calculated for uniform random copolymers with $u_{\mathrm{M}}=1.1$ and $v=0.007 .[\alpha]_{\mathrm{m}}=$ $60010^{-1} \mathrm{deg} \mathrm{cm}^{2} \mathrm{~g}^{-1}$.

reversal in ten chains of $N=30$, i.e., $n_{\mathrm{R}}=0.1$ and the value of 1.1 for $u_{\mathrm{M}}$ suggests that NIC is a moderately strong sergeant. The data at $-20^{\circ} \mathrm{C}$ scatter too much to allow a similar analysis, but appear to be characterized by a smaller $v$. It is necessary to have more detailed data to claim higher accuracies in $u_{\mathrm{M}}$ and $v$.

The symmetry of helix sense in enantiomorphous copolymers of isocyanate may be broken by the excess presence of one enantiomer, giving rise to the optical activity. Green et al $^{25}$ made an interesting finding that $[\alpha]_{\mathrm{D}}$ of the random copolymer of $(R)$ - and $(S)-2,6-$ dimethylheptyl isocyanates changed disproportionately with the mole fraction $2 x-1$ of excess $(R)-2,6-\mathrm{di}-$ methylheptyl isocyanate. This is a copolymer of type 1 , which may be modeled by taking $u_{\mathrm{M}, \mathrm{R}}=1 / u_{\mathrm{M} . \mathrm{S}}=u_{\mathrm{M}}$ and $v_{\mathrm{R}}=v_{\mathrm{S}}=v$. Figure 7 compares their data for $[\alpha]_{\mathrm{D}}$ in chloroform at $25^{\circ} \mathrm{C}$ (circles) with the theoretical values calculated for this model; $[\alpha]_{\mathrm{m}}$ for PNIC is assumed to be $60010^{-1} \mathrm{deg} \mathrm{cm}^{2} \mathrm{~g}^{-1}$. The data can be fitted to the curve with $u_{\mathrm{M}}=1.1$ and $v=0.007$; the accuracies of these values are only moderate. We see that the values of $u_{\mathrm{M}}$ and $v$ of $(R)$-2,6-dimethylheptyl isocyanate residue are nearly the same in the NIC-HIC copolymers and in the $R-S$ copolymers. Thus to a first approximation, the helix stability of $(R)$-2,6-dimethylheptyl isocyanate does not depend on its location on the polymer chain. It must be noted however that this conclusion must be taken with reservation in quantitative terms. This is because the data analyzed are for as-polymerized samples of unknown heterogeneity in molecular weight and mono-

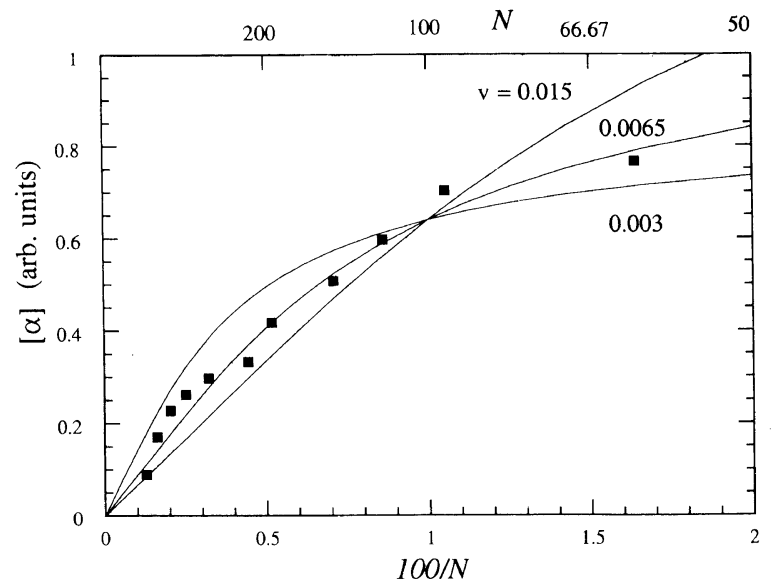

Figure 8. Molecular weight dependence of $[\alpha]$ for chirally initiated poly(butyl isocyanate) in chloroform at $25^{\circ} \mathrm{C}$. Squares, experimental data of Okamoto et al. ${ }^{26}$; curves, theoretical values calculated using eq 16 with the indicated parameter values without considering the end effect. $[\alpha]_{\mathrm{m}}(\alpha-1) /(\alpha+1)$ is adjusted as to give $[\alpha]$ of 0.64 at $N=100$.

mer composition. Our experience ${ }^{33,34}$ suggests that the molecular weight polydispersity has a profound effect on $[\alpha]$ even for a sufficiently high molecular weight sample and $[\alpha]_{\mathrm{m}}$ may depend on composition (see the previous footnote). As shown in Table I, our results for $u_{\mathrm{M}}$ and $v$ are compared with those of Selinger and Selinger. ${ }^{32}$

Figure 8 shows optical rotation data of Okamoto et al. $^{26}$ for chirally initiated poly(butyl isocyanate) (PBI/Li-MMP). In this case the initiator fragment prefers the right-handed helix, with its effect being decreasing rapidly with increasing $N$. This change in $[\alpha]$ for $N$ larger than 60 is described almost quantitatively by taking $[\alpha]_{\mathrm{m}}(a-1) /(a+1)=1.14$ and $v=0.0065$ (curve by eq 9 with eq 16$) ;[\alpha]_{\mathrm{m}}$ and $a$ cannot be estimated separately because no data is available for $[\alpha]_{\mathrm{m}}$. This $v$ value happens to agree with that for Green's polymer obtained above and is favorably compared with that found for deuterated poly(hexyl isocyanate)s. ${ }^{33,34}$ The data for $N<50$ (not shown here) is shown to deviate significantly above the solid curve for the reason as yet unknown to us.

Figure 9 shows a comparison between experiment and theory for chirally initiated poly $(m$-methylphenyl isocyanate) (PmMePI/Li-MMP). ${ }^{27}$ In the panel (a), theoretical curves for different $v$ values are drawn to approximately follow the data points by choosing appropriate values for $[\alpha]_{\mathrm{m}}(a-1) /(a+1)$. The data for $N$ larger than 13 can be fitted accurately to the theory 


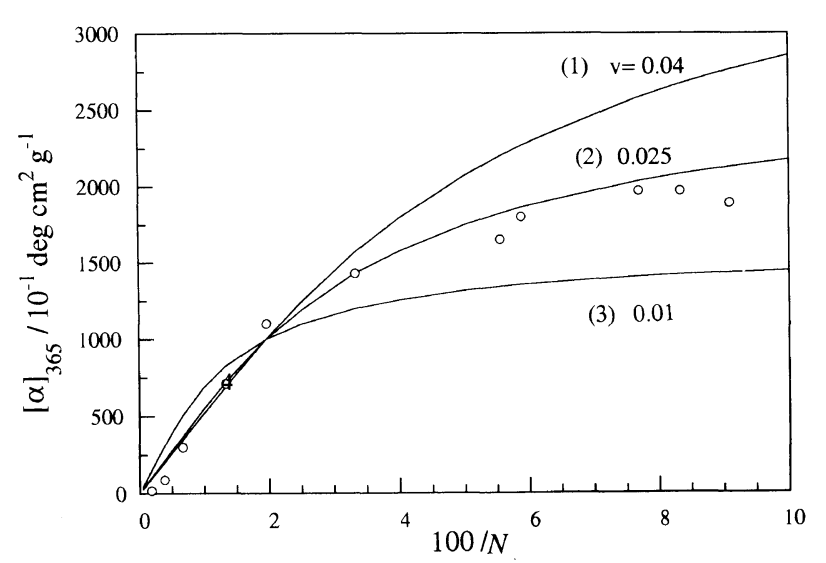

(a)

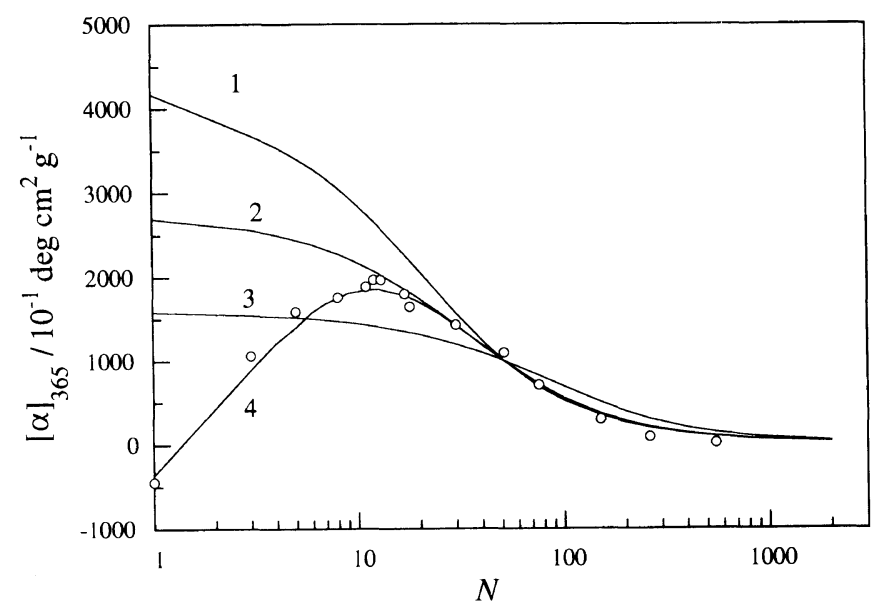

(b)

Figure 9. $N$ dependence of $[\alpha]$ for a chirally initiated $\operatorname{poly}(m-$ methylphenyl isocyanate) in tetrahydrofulan at $25^{\circ} \mathrm{C}$ : circles, data of Maeda et al. ${ }^{27}$ Panel (a): curves $1-3$, theoretical values calculated using eq 16 for the indicated $v$ values, with $[(a-1) /(a+1)][\alpha]_{\mathrm{m}}$ being adjusted to 1070 at $N=51$ for comparison; panel (b): curves $1-3$, theoretical values calculated with the same parameter values as in panel (a); curve 4 , theoretical values calculated using eq 16 along with eq 22 with the parameter values: $a=6, b=1, v=0.025, k=0.2,[D]=-900$

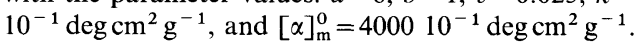

if the value of $v$ is taken to be $0.025 \pm 0.01$, more than twice as large as that for poly(NIC-HIC). Furthermore $a$ is calculated to be 9 if $[\alpha]_{\mathrm{m}}$ is assumed to be 3560 $10^{-1} \mathrm{deg} \mathrm{cm}^{2} \mathrm{~g}^{-1}$; this $a$ valve corresponds to $\Delta G$ of $1.3 \mathrm{kcal} \mathrm{mol}^{-1}$ when expressed as $a=\exp (-\Delta G / R T)$. A quantum mechanical estimate of $a$ may be possible from the known chemical structure of the chain end. ${ }^{35}$ The values of $n_{\mathrm{R}}$ calculated with the parameters values employed showed that the occurrence of helix reversal cannot be negligible even at $N$ smaller than 50 and indicate that the $N$ dependence arises predominantly from the helix reversal.

The data points at $N$ smaller than 10 show remarkable downswing (see panel b), which cannot be explained by the present theory. For this polymer, $[\alpha]$ should start at $N=0$ with a small negative value, which is due to the initiator fragment. However this fragment forces the polyisocyanate chain to take a right-handed helical conformation, yielding positive $[\alpha]$. Initially $[\alpha]$ increases with increasing $N$, and there would exist no helix reversal for such short chains. We propose the following hypothesis to account for this characteristic behavior.
In the above treatment, $[\alpha]_{\mathrm{m}}$ is defined as the specific rotation of monomer residue in a perfect helical conformation and is assumed not to depend on chain length. This assumption may not be correct for chains with only a few monomer residues and this is actually the case with $\alpha$-helical polypeptides, where $[\alpha]_{\mathrm{m}}$ increases asymptotically with chain length. ${ }^{36-38}$ We follow these studies to assume that $[\alpha]_{\mathrm{m}}$ is given by

$$
[\alpha]_{\mathrm{m}}=[\alpha]_{\mathrm{m}}^{0}[1-\exp (-k N)]
$$

where $[\alpha]_{\mathrm{m}}^{0}$ is $[\alpha]_{\mathrm{m}}$ for an infinitely long chain and $k$ is a numerical constant. Thus in this refinement we have four adjustable parameters $a, v,[\alpha]_{\mathrm{m}}^{0}$, and $k$. It is also probable that the chiral fragment contributes to specific rotation as $[D]$. Taking these considerations into account, we may express $[\alpha]$ in the following form:

$$
[\alpha]=\left(2 f_{\mathrm{M}}-1\right)[\alpha]_{\mathrm{m}}+[D] / N
$$

Trial-and-error procedures were repeated with possible values for the parameters $[\alpha]_{\mathrm{m}}^{0}, k$, and $[D]$ with the above $a$ and $v$ values assumed, to obtain the best agreement between experiment and theory. Curve 4 in panel (b) of Figure 9 represents the theoretical values with the optimal parameter values thus determined, which is seen to reproduce the data points almost quantitatively. Therefore we conclude that consideration of the $N$ dependence of $[\alpha]_{\mathrm{m}}$ is one of reasonable explanations of the characteristic optical rotation behavior of this polymer, although no theoretical justification of eq 22 is found at present.

Okamoto and collaborators ${ }^{26,27}$ found significant temperature dependence of $[\alpha]$ for the two polymers discussed in Figures 8 and 9. For PmMeP/Li-MMI the temperature dependence (relative to that at $25^{\circ} \mathrm{C}$ ) was much larger for $N=198$ than for $N=3$. When compared at similar $N$, PmMeP/LI-MMI showed more pronounced temperature dependence than PBI/Li-MMI. These features were explained qualitatively by theoretical calculations using the above values of $v$ with a constant value for $\Delta G_{\mathrm{r}}$. Indeed it was possible to reproduce these results with appropriate temperature dependence incorporated in $a$ and $v$. However there is no sufficient data for such a quantitative analysis.

\section{CONCLUDING REMARKS}

As found with chiral polyisocyanates, the optical activity of the polyisocyanates examined here is shown to originate from helical conformation with occasional helix reversals. Theoretically the optical activity is determined by the three parameters: the optical rotation for perfect helix $[\alpha]_{\mathrm{m}}$, the free energy preference of a particular helix sense, $\Delta G_{\mathrm{h}}$, and the helix reversal energy $\Delta G_{\mathrm{r}}$. All the numerical results obtained are summarized in Table I in comparison with those for partially deuterated poly(hexyl isocyanates). ${ }^{33,34}$

For random copolymers of chiral and achiral isocyanates, the optical rotation as a function of molecular weight and monomer composition was consistently described using apprpriate values of $\Delta G_{\mathrm{h}}$ (small) and $\Delta G_{\mathrm{r}}$ (large, $2730 \mathrm{cal} \mathrm{mol}^{-1}$ ). The value of 1.1 for $u_{\mathrm{M}}$ is translated into $\Delta G_{\mathrm{h}}$ of $113 \mathrm{cal} \mathrm{mol}^{-1}$ at $25^{\circ} \mathrm{C}$; namely right-handed helix is preferred over the left-handed helix 
to the extent corresponding to this energy for $(R)-2,6-$ dimethylheptyl isocyanate residue. For achiral polyisocyanates prepared with chiral initiators, the optical rotation is due to the preference of a particular helix sense enforced by the chiral initiator fragment attached on the chain end but the molecular weight dependence of optical rotation is essentially determined by helix reversal. The helix reversal probability is larger for the aromatic polyisocyanate compared with alkyl polyisocyanates. This may be related to possible difference in conformation of the backbone amide groups between the two polyisocyanates, namely, the partial doublebond nature may be weaker for poly $(m$-methylphenyl isocyanate) compared with the butyl polymer due to conjugation between amide bonds and phenyl rings. It is interesting to examine how this is related to the global conformation of the polymer, e.g., its stiffness. The present result confirms quantitatively Okamoto et al.'s argument ${ }^{26,27}$ that the molecular weight dependence of $[\alpha]$ comes from helix reversal. However the numerical results obtained in the present study have to be taken with some reservation, because these values have been estimated from the experimental data for as-polymerized samples of unknown polydispersity, which may not be accurate to enough for a quantitative discussion.

Acknowledgments. The work at Osaka University was supported by the Grant-in-Aid for Scientific Research from the Ministry of Education, Science, Sports and Culture of Japan. The work at the Polytechnic University was supported by the Chemistry and Materials Divisions of the National Science Foundation and by the Petroleum Research Fund, administered by the AmericanChemical Society. Thanks are due to Professor Yoshio Okamoto of Nagoya University for valuable discussions on type 2 polymers.

\section{REFERENCES}

1. U. Shmueli, W. Traub, and K. Rosenheck, J. Polym. Sci. A-2, 7, 515-524 (1969).

2. T. C. Troxell and H. A. Scheraga, Macromolecules, 4, 528 (1971).

3. C. C. Han and H. Yu, Polym. Prepr., Am. Chem. Soc., Div. Polym. Chem., 14, 121 (1973).

4. A. E. Tonelli, Macromolecules, 7, 854 (1974).

5. M. L. Mansfield, Macromolecules, 19, 854 (1986).

6. R. C. Cook, Macromolecules, 20, 1961 (1987).

7. R. C. Cook, R. D. Johnson, C. G. Wade, B. Muñoz, and M. M. Green, Macromolecules, 23, 3454 (1990).
8. M. M. Green, R. A. Gross, R. C. Cook, and F. C. Schilling, Macromolecules, 20, 2636 (1987).

9. A. J. Bur and L. J. Fetters, Chem. Rev., 76, 727 (1976).

10. S. Lifson, C. E. Felder, and M. M. Green, Macromolecules, 26, 4142 (1992).

11. M. N. Berger and B. M. Tidswell, J. Polym. Sci., Polym. Symp., 42, 1063 (1973).

12. D. N. Rubingh and H. Yu, Macromolecules, 9, 681 (1976).

13. H. Murakami, T. Norisuye, and H. Fujita, Macromolecules, 13 , 345 (1980).

14. M. Kuwata, H. Murakami, T. Norisuye, and H. Fujita, Macromolecules, 17, 2731 (1984).

15. T. Norisuye and H. Fujita, Polym. J., 14, 143 (1982).

16. T. Itou, H. Chikiri, A. Teramoto, and S. M. Aharoni, Polym. J., 20, 143 (1988).

17. T. Norisuye, in "The Eleventh Taniguchi Conference," SpringerVerlag, Hakone, Japan, 1989, p 178.

18. S. Takada, T. Itou, H. Chikiri, Y. Einaga, and A. Teramoto, Macromolecules, 22, 973 (1989).

19. M. M. Green, C. Andreola, B. Muñoz, M. P. Reidy, and K. Zero, J. Am. Chem. Soc., 110, 4063 (1988).

20. M. M. Green, M. P. Reidy, R. D. Johnson, G. Darling, D. J. O'Leary, and G. Wilson, J. Am. Chem. Soc., 111, 6452 (1989).

21. S. Lifson, C. Andreola, N. C. Peterson, and M. M. Green, J. Am. Chem. Soc., 111, 8850 (1989).

22. C. Andreola, Thesis, Polytechnic University (1991).

23. M. M. Green, S. Lifson, and A. Teramoto, Chirality, 3, 285 (1991).

24. M. M. Green, N. C. Peterson, T. Sato, A. Teramoto, R. Cook and S. Lifson, Science, 268, 1860 (1995).

25. M. M. Green, B. A. Garetz, B. Muñoz, HePing Chang, S. Hoke and R. G. Cook, J. Am. Chem. Soc., 117, 4181 (1995).

26. Y. Okamoto, M. Matsuda, T. Nakano, and E. Yashima, Polym. J., 25, 391 (1993).

27. K. Maeda, M. Matsuda, T. Nakano, and Y. Okamoto, Polym. J., 27, 141 (1995).

28. D. Poland and H. A. Scheraga, "Theory of Helix-Coil Transitions in Biopolymers," Academic Press, New York, N.Y., 1970.

29. A. Teramoto and H. Fujita, Adv. Polym. Sci., 18, 65 (1975).

30. A. Teramoto and H. Fujita, J. Macromol. Sci., Rev. Macromol. Chem., C15, 165 (1976).

31. H. Gu, A. Teramoto, L. Varichon, and M. M. Green, Polym. Prepr., Jpn., 44, 3555 (1995).

32. J. V. Selinger and R. L. B. Selinger, Phys. Rev. Lett., 76, 58 (1996).

33. H. Gu, Y. Nakamura, T. Sato, A. Teramoto, M. M. Green, C. Andreola, N. C. Peterson, and S. Lifson, Macromolecules, 28 , 1016 (1995).

34. N. Okamoto, F. Mukaida, H. Gu, Y. Nakamura, T. Sato, A. Teramoto, M. M. Green, C. Andreola, and N. C. Peterson, Macromolecules, 29, 2878 (1996).

35. Suggestion of Professor Yoshio Okamoto of Nagoya University.

36. R. W. Woody and I. Tinoco, Jr., J. Chem. Phys., 46, 4927 (1967).

37. J. N. Vournakis, J. F. Yan, and H. A. Scheraga, Biopolymers, 6, 1531 (1968).

38. T. Matsumoto and A. Teramoto, Biopolymers, 13, 147 (1975). 